\title{
From Star Formation to Compact Remnants: X-ray Studies of Spiral Galaxies
}

\author{
Roberto Soria, Kinwah Wu \\ MSSL, University College London, UK
}

\begin{abstract}
High-resolution multiwavelength studies of nearby galaxies are essential to understand the full cycle of star formation, and the balance between gas recycling and creation of compact objects. In particular, we study this cycle in the environment of ultra-luminous X-ray sources.
\end{abstract}

\section{Multiwavelength studies of spiral galaxies}

X-ray properties of spiral galaxies reveal their current activity and star formation history. In our studies of nearby galaxies with Chandra and XMM-Newton (Soria 2002; Soria \& Wu 2003; Soria, Pian \& Mazzali 2004), we have used Xray colour-colour diagrams to identify different classes of sources: supersoft; medium-soft (mostly X-ray supernova remnants); X-ray binaries in a soft or hard state (Fig. 1, left panel). The face-on, late-type spiral M 83 (distance $\approx 4$ $\mathrm{Mpc}$ ) is a particularly suitable test case: it shows "normal" star formation along the grand-design spiral arms, and a starburst nucleus.

Most of the unresolved diffuse emission in M 83 is due to hot thermal plasma at $k T \approx 0.2-0.6 \mathrm{keV}$ along the spiral arms, and $\approx 0.3-0.8 \mathrm{keV}$ in the nuclear region. Studying its temperature variations, and spatial correlations with $\mathrm{H} \alpha$ (Fig. 1, right panel) and radio emission, we can probe the most recent star formation. Slope and breaks in the cumulative luminosity distribution of the discrete sources probe star formation over longer timescales $\left(\sim 10^{9} \mathrm{yr}\right)$.

\section{Gas, young stars, and ultra-luminous sources}

Accreting compact objects with X-ray luminosities up to $\approx 10^{40} \mathrm{erg} \mathrm{s}^{-1}$ (ULXs) have been found in many star-forming galaxies; they may be accreting black holes with masses $\gg 10 M_{\odot}($ eg, Colbert \& Mushotzky 1999). Optical studies of their environments (Pakull \& Mirioni 2002) have revealed that they are often located inside H II regions (Fig. 2) or shell-like, expanding X-ray ionised nebulae, with diameters of a few hundred pc. We are currently studying the link between the nebulae, the compact objects and their young, massive donor stars, and what environmental conditions (eg, metal abundance) are more favourable to the formation of intermediate-mass black holes in young clusters. 

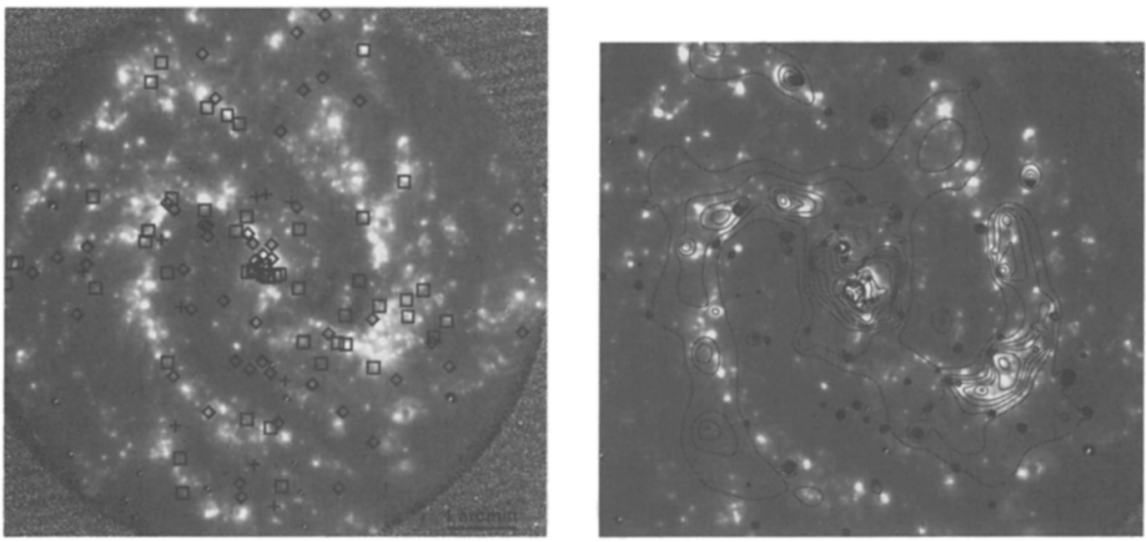

Figure 1. Left: discrete X-ray sources in M 83, plotted on an $\mathrm{H} \alpha$ map. Squares are (soft) SNR candidates, mostly associated with H II regions; diamonds are likely X-ray binaries; crosses are supersoft sources. Right: different gas phases can be investigated from correlations between diffuse X-ray emission (contours) and $\mathrm{H} \alpha$ emission (greyscale).

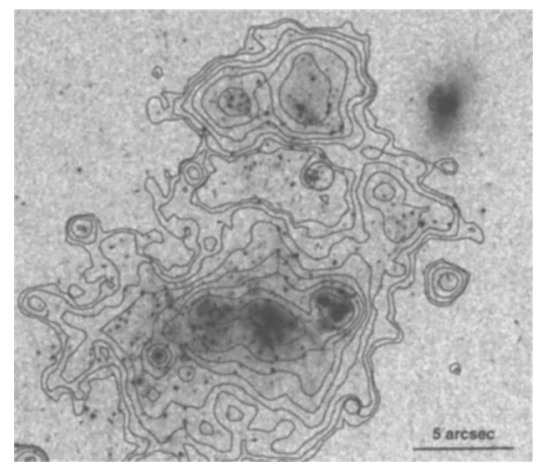

Figure 2. A ULX $\left(L_{x} \approx 2 \times 10^{40} \mathrm{erg} \mathrm{s}^{-1}\right)$ in NGC 4559, located inside a shell-like $\mathrm{H}$ II region. $\mathrm{H} \alpha$ contours and Chandra error circle are plotted on an HST/WFPC2 f555w greyscale map.

\section{References}

Colbert, E. J. M., \& Mushotzky, R. F. 1999, ApJ, 519, 89

Pakull, M. W., \& Mirioni, L. 2002, proceedings of the symposium "New Visions of the X-ray Universe", ESTEC 2001 (astro-ph/0202488)

Soria, R. 2002, proceedings of the IAU Symposium No.214 (astro-ph/0211024)

Soria, R., \& Wu, K. 2003, A\&A, 410, 53

Soria, R., Pian, E., \& Mazzali, P. 2004, A\&A, 413, 107 